\title{
Endometriosis: Correlation of Severity of Pain with Stages of Disease
}

\author{
TS CHOWDHURY ${ }^{\mathrm{a}}$, N MAHMUD ${ }^{\mathrm{b}}, \mathrm{TACHOWDHURY}^{\mathrm{c}}$
}

\begin{abstract}
Summary:
Objective: To assess the prevalence and severity of pain in patients with endometriosis and to evaluate the relationship between the severity of pelvic pain with different stages of endometriosis.
\end{abstract}

Methods: A prospective observational study conducted among 65 patients diagnosed with endometriosis during surgery in Infertility Management Center Dhaka, a specialized center for treatment of infertility and assisted reproductive technologies from January 2008 to January 2009.

Result: Majority of the patients were between 26-30 years. Pelvic pain was the predominant symptom in $78.5 \%$ patients and the rest were asymptomatic. Among the symptomatic patients, most common symptom was dysmenorrhoea $(n=47$, 92.1\%). When severity of pain was graded; it was observed that most (37\%) presented with moderate pain. Infertility was present in $85 \%$ patients, mostly ( $n-47=74 \%)$ in primary sub fertility group. The commonest site of endometriosis was

Introduction:

Endometriosis is defined as the presence of endometriallike tissue outside the uterus, which induces a chronic, inflammatory reaction. ${ }^{1}$ The exact prevalence of endometriosis estimates approximately $10 \%$ within the general population. ${ }^{2}$

Endometriosis diagnosis is based on the women's history, symptoms and signs. While some women with endometriosis experience painful symptoms and/or infertility, others have no symptoms at all. And definitive criteria to determine that the pain is actually caused by

a. Dr. Tanzeem S Chowdhury, Assistant Professor, Dept. of Obs. and Gynae, BIRDEM General Hospital and Ibrahim medical college, Dhaka.

b. Dr. Nusrat Mahmud, Consultant, Center for Assisted Reproduction, BIRDEM, Dhaka.

c. Prof. TA Chowdhury, Chief Consultant and Professor, Dept. of Obs and Gynae, BIRDEM General Hospital and Ibrahim medical college, Dhaka.

Address of Correspondence: Dr Tanzeem S Chowdhury, Assistant Professor, Obs. \& Gynae, BIRDEM, Dhaka. E-mail: tanzeemsc@gmail.com. Mobile: 01713037853

Received: 18 October, 2014

Accepted: 18 April, 2016 uterosacral ligament ( $\mathrm{n}-\mathbf{5 8}=\mathbf{8 9 \%}$ ) and ovarian endometriosis is noted in (63\%) cases. Most of the lesions (35\%) were black, haemosiderin deposits. When r-ASRM staging system was applied, majority (58.4\%) of the patients was in stage IV disease and most of the patients (31\%) with endometrioma were in stage IV disease. The study revealed a strong positive correlation between severe pain and stage IV disease (Correlation co efficient 0.711). Moderate forms of pain and severity of disease did not show any positive correlation in this study (Correlation co efficient 0.390). There was negative correlation between milder forms of pain with severity of disease.

Conclusion: There was no relationship between frequency and severity of pain symptoms and disease stage of endometriosis.

Key words: Endometriosis, r-ASRM score, visual analogue scale.

(J Bangladesh Coll Phys Surg 2016; 34: 135-139)

endometriosis is lacking. So, the nature of the relationship between chronic pain and endometriosis remains poorly understood. This results in many women receiving either delayed or suboptimal care. ${ }^{1}$

Laparoscopy or laparotomy allows staging of endometriosis according to the r-ASRM classification system (Revised American Society for Reproductive Medicine classification of endometriosis: 1996, 1997). This classification system assigns points to the different locations of the disease thus resulting in four stages: minimal, mild, moderate and severe.

The aim of this study is to assess the prevalence and severity of pain in patients diagnosed with endometriosis during laparoscopy or laparotomy and to evaluate the relationship between the severities of pelvic pain with different stages of endometriosis.

\section{Method}

This study was done in Infertility Management Center Dhaka, a specialized center for treatment of infertility and Assisted reproductive technologies. This study was conducted from January 2008 to January 2009. It was a prospective observational study. At first 112 
women with regular menstrual cycles were recruited who underwent laparoscopy or laparotomy due to various reasons. Before surgery, each patient completed a questionnaire regarding age, pelvis pain and infertility if present. Severity of pain was graded according to visual analogue scale, 0-4 mild pain, 5-7 moderate pain, 8-10severe state like incapacitation or requiring strong analgesic. Then these patients underwent laparoscopy or laparotomy according to decision previously taken. Amongst them, 65 patients with diagnosis of endometriosis during surgery were included in this study. Forty seven patients were excluded from the study as they had pain due to PID, post operative adhesions and or genital malformation. Patients who were treated for pelvic pain and endometriosis in the previous six months other than NSAID like GnRh analogues/ OCPs were also excluded from the study. During the surgical procedure all the visible lesions were noted and staged according to r-ASRM classification. In addition, color, site of lesion and presence of endometrioma were also noted.

\begin{tabular}{|c|c|c|c|c|c|c|c|c|c|c|c|}
\hline & 0 & 1 & 2 & 3 & 4 & 5 & 6 & 7 & 8 & 9 & 10 \\
\hline & $\begin{array}{l}\text { శ్ } \\
\text { ฉ } \\
\stackrel{z}{2}\end{array}$ & 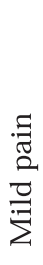 & 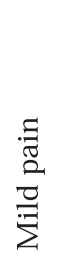 & 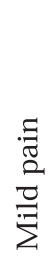 & 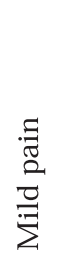 & 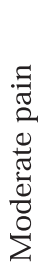 & 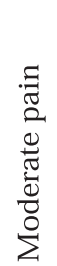 & 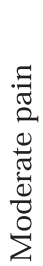 & 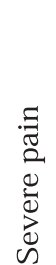 & 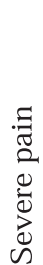 & 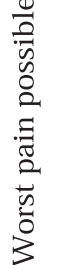 \\
\hline 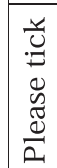 & & & & & & & & & & & \\
\hline
\end{tabular}

Fig -1: Visual Analogue Pain scale

\section{Results:}

Total 65 patients with regular menstrual cycles who were diagnosed with endometriosis during surgery were included in this study and the following results were found.

Majority of the patient population belonged to 26-30 years of age range (Table-I). Pelvic pain was the predominant symptom in $78.5 \%$ patient population and $21.5 \%$ patients were asymptomatic.
Table-I

$$
\begin{gathered}
\text { Incidence of endometriosis according to age } \\
\text { distribution }(n=65)
\end{gathered}
$$

\begin{tabular}{lcc} 
Age group & Number of patients & Percentage (\%) \\
\hline Less than 20 & 2 & 3 \\
21 to 25 & 15 & 23 \\
26 to 30 & 26 & 40 \\
31 to 35 & 16 & 25 \\
36 to 40 & 6 & 9 \\
\hline Total & 65 & 100 \\
\hline
\end{tabular}

Among the symptomatic patients, 47 (92.1\%) had dysmenorrhea, 19 (37.2\%) patients had chronic pelvic pain, deep dyspareunia in 11 (21.6\%), dyschezia in 11 (21.6\%) and dysuria in 7 (13.7\%) patients.

Severity of pain was graded according to the visual analogue pain scale. We observed $30 \%$ patients experienced mild form of pain, $37 \%$ with moderate pain and $33 \%$ with severe form of pain.

Infertility was the predominant cause in $85 \%$ patients and remaining $15 \%$ did not have problem regarding fertility. Those patients who suffered from infertility were mostly (n- $47=74 \%$ ) in the primary group and 14 patients (26\%) suffered from secondary sub fertility.

Depending on the disease pattern, 50 patients (77\%) underwent laparoscopy and the remaining 15 (23\%) patients had undergone laparotomy.

During the operative procedure the commonest site of endometriosis was found in uterosacral ligament (n$58=89 \%$ ), ovarian endometriosis is noted in (63\%) cases. The other sites were rectovaginal septum, broad ligament, peritoneum and fallopian tubes as shown in Table II

\section{Table-II}

Distribution of endometriosis ( $n=65)$

\begin{tabular}{lcc} 
Site & $\begin{array}{c}\text { Number of } \\
\text { patients }\end{array}$ & Percentage (\%) \\
\hline Uterosacral ligament & 58 & 89.2 \\
Ovaries & 41 & 63.0 \\
Recto vaginal septum & 21 & 32.3 \\
Broad ligament & 25 & 38.4 \\
Peritoneum & 12 & 18.4 \\
Fallopian tube & 9 & 13.8 \\
\hline
\end{tabular}


Regarding the type of endometriotic lesion, it was observed that most of the lesions (35\%) were black, haemosiderin deposits followed by red, pink and vesicular blobs (Table - III)

\section{Table-III}

Type of endometriotic lesion $(n=65)$

\begin{tabular}{lcc} 
Type & $\begin{array}{c}\text { Number of } \\
\text { patient }\end{array}$ & $\begin{array}{c}\text { Percentage } \\
\text { (\%) }\end{array}$ \\
\hline Black, & 23 & $35.38 \%$
\end{tabular}

haemosiderin deposits

Red, mild pink, flame like, 16 $24.61 \%$ vesicular blobs, clearvesicles

White, peritoneal defects, $12 \quad 18.46 \%$ yellow brown

No specific type $\quad 14 \quad 21.53 \%$

Figure 2 showed the distribution of patients according to the stage of endometriosis (r-ASRM). Majority (58.4\%) of patients were in stage IV endometriosis.

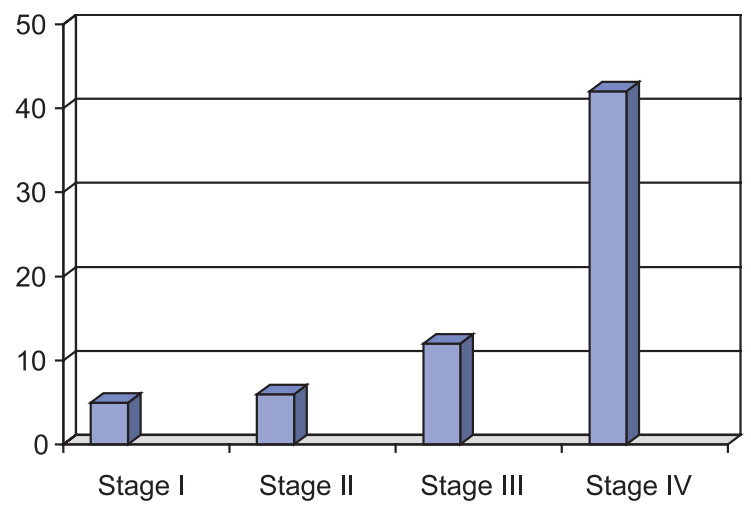

Fig.-2: Stages of endometriosis according to R-AFS score $(n=65)$

When presence or absence of endometrioma was looked for $(n=41)$ among the patients with ovarian endometriosis, 31\% patients with endometrioma were in stage IV endometriosis. Patients with stage I disease did not show any evidence of endometrioma (Fig 3). This reflected that endometrioma was usually not present in the milder forms of endometriosis (stage I and II)

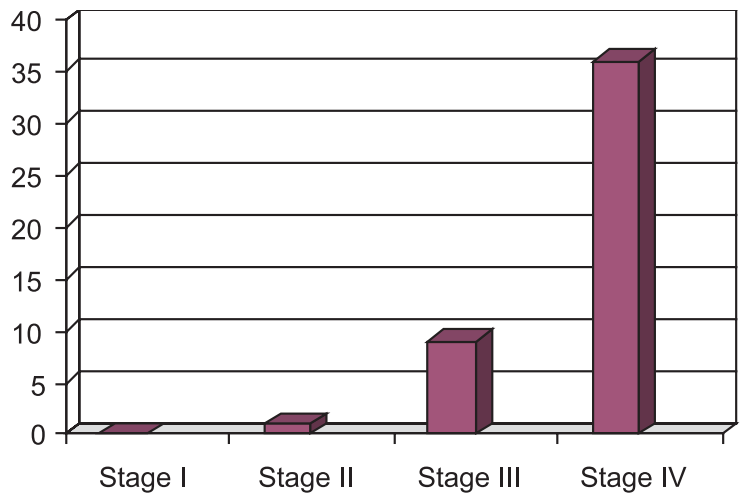

Fig.-3: Stages of endometriosis according to R-AFS score $(n=65)$

Table-IV showed the correlation of pain score with rAFS scores and it showed strong positive correlation between severe pain and stage IV disease (Correlation co efficient 0.711 ). Moderate forms of pain and severity of disease did not show any positive correlation in this study (Correlation co efficient 0.390 ). There was in fact negative correlation between milder forms of pain with severity of disease.

Table-IV

Correlation of pain score with $R$-AFS score $(n=65)$

\begin{tabular}{lcccc} 
Pain & $\begin{array}{c}\text { Stage I } \\
\text { Minimal }\end{array}$ & $\begin{array}{c}\text { Stage II } \\
\text { Mild }\end{array}$ & $\begin{array}{c}\text { Stage III } \\
\text { Moderate }\end{array}$ & $\begin{array}{c}\text { Stage IV } \\
\text { Severe }\end{array}$ \\
& 5 & 6 & 12 & 42 \\
\hline No pain $\mathrm{n}=14$ & 2 & 2 & 2 & 8 \\
Mild $\mathrm{n}=15$ & 2 & 2 & 4 & 7 \\
Moderate $\mathrm{n}=19$ & 1 & 2 & 4 & 12 \\
Severe $\mathrm{n}=17$ & 0 & 0 & 2 & 15 \\
$\begin{array}{l}\text { Correlation } \\
\text { efficient }\end{array}$ & -0.225 & -0.225 & 0.390 & 0.711 \\
\hline
\end{tabular}

\section{Discussion:}

In this study, most of the patients were 26-30 years old. A serious challenge in endometriosis diagnosis is that even though the age of onset of menstrual pain is early, there is a significant delay of $7-11$ years ${ }^{1,3}$ before the average woman is diagnosed. Propst and Yeung in two different studies also showed that among adolescents who complained of dysmenorrhea, approximately $70 \%$ eventually were diagnosed with endometriosis, so this symptom warrants special attention in young women. ${ }^{4,5}$ 
This study also revealed that even though pelvic pain was predominant in most patients, about $22 \%$ patients were asymptomatic. This was also seen in a study done by Porpora in 1999 , which showed that $11.1 \%$ woman had no symptom of pelvic pain even though they had biopsy proven endometriosis. ${ }^{6}$

Pelvic symptoms like dysmenorrhea, chronic pelvic pain, deep dyspareunia, cyclical intestinal complaints, fatigue/ weariness and infertility continue to be the leading symptoms of endometriosis. In this study the predominant symptom was dysmenorrhea (92\%) followed by chronic pelvic pain (37\%) and dyspareunia (21\%). In one cross-sectional survey carried out in 12 tertiary care centers in 10 countries in 2013 also showed that dysmenorrhea (59\%) and chronic pelvic pain (60\%) were the predominate symptoms of endometriosis. 7 Previously in a different study, Forman also concluded that dysmenorrhea was the only symptom significantly predictive of endometriosis. ${ }^{8}$ No differences in the rates of pelvic pain, dyspareunia or vaginal discharge were seen among women with endometriosis, compared to those with normal pelvis or adhesions. ${ }^{8}$ In another prospective study done by Eskenaze et al in Italy, where women scheduled to undergo various gynecological operations were interviewed concerning infertility, dysmenorrhea, dyspareunia and non-cyclical pelvic pain, none of these were predictive of the diagnosis of endometriosis. ${ }^{9}$ However, women eventually surgically diagnosed with endometriosis reported more intensive dysmenorrhea than those with no diagnosis of endometriosis. ${ }^{9,10}$

In this study, we observed a strong correlation of endometriosis with infertility, mostly primary infertility. An association between endometriosis and infertility has long been noted. The prevalence of endometriosis was 47\% (104/221), including stage I (39\%, 41/104), stage II (24\%, 25/104), stage III (14\%, 15/104), and stage IV (23\%, 23/104) disease. ${ }^{11}$ Multiple other studies, the majority of which were retrospective, also indicated that the monthly fecundity of patients with endometriosis may be decreased by half compared to women without the disease. The precise cause-effect relationship between endometriosis and infertility remains controversial. In advanced cases of endometriosis, with distorted pelvic anatomy, the mechanism of infertility is more easily explained, but there is no satisfactory hypothesis that unequivocally explains the association of early stages of endometriosis with infertility. Endocrine dysfunctions such as luteal phase defect and luteinized unruptured follicle syndrome have been proposed as the cause in many studies. ${ }^{12}$

Laparoscopy with or without histological verification is widely used to diagnose and rule out the presence of endometriosis. Furthermore r-ASRM classification can be used to express the severity of the disease. However, the literature on the diagnostic value of a laparoscopy is very limited. Data on complications and adverse events are similarly limited, a reporting bias. A negative diagnostic laparoscopy in women with symptoms and signs of the disease is highly reliable for the exclusion of the diagnosis of endometriosis. ${ }^{13}$

In this study 50 patients underwent laparoscopy and the rest (15) underwent laparotomy according to the decision taken previously. The commonest site of endometriosis was uterosacral ligament (n-58=89\%) and ovarian endometriosis was noted in (63\%) cases. But Vercellini et al conducted a study on 244 symptomatic patients and found out that ovarian endometriosis was the commonest site and found in 108 patients followed by combined peritoneal and ovarian lesion in 57 patients. $^{14}$

It was also observed in this study that that most of the lesions (35\%) were black, haemosiderin deposits followed by red, pink, vesicular blobs in 25\% cases. There were also vesicular blobs, clear vesicles and white or yellow peritoneal defects. There were even spots of endometriosis which did not fall in any specific type. (21\%).This is of immense clinical importance. Because of the variation in presentation of endometriosis, it is often very difficult to diagnose the condition. Precise diagnosis regarding presence, location, and extent of endometriosis is very much necessary for further evaluation and surgical planning of endometriosis.

This study revealed that endometrioma was present in $31 \%$ patients mostly in stage IV endometriosis. This reflected that endometrioma was usually a feature of severe disease and that isolated endometrioma was unlikely without the involvement of the peritoneal cavity.

After noting the site, type and extent of the disease, the r-ASRM scoring was done and it was correlated with the pain score given preoperatively. It showed that there was a strong positive correlation between severe pain and stage IV disease (Correlation co efficient 0.711). There was no correlation between moderate pain and severity of disease (Correlation co efficient 0.390 ). There is a negative correlation between milder forms of pain with severity of disease. Study conducted by Vercellini also concluded that stage of endometriosis per se, 
independent of the lesion site, was not correlated with the frequency or severity of dysmenorrhea and nonmenstrual pain. The severity of deep dyspareunia was found to be inversely proportional to the endometriosis score. ${ }^{14}$ Another study by Porpora et al. similarly concluded that no significant correlation was found among revised American Fertility Society stage of endometriosis; presence and size of ovarian endometriomas; extent, type, and site of peritoneal lesions; and pain scores. ${ }^{15}$ This is in contrast to a study done by Mehmud in Pakistan in 2007 which showed there was a significant positive correlation between chronic pelvic pain and R-AFS scoring but not with dysmenorrhea. ${ }^{16}$

So, this study further supported that in cases of pelvic endometriosis, a newer classification is needed as a supplement to the $\mathrm{r}$ - ASRM score. Enzian classification was first described by Haas et al ${ }^{17}$ in 2013 which revealed that the locations of endometriosis in the Enzian classification correlated partially with clinical symptoms, and the classification's severity grades correlated substantially with pain and dysmenorrhea.

Small sample size of this study does not depict the varied picture of endometriosis. This is the limitation of this study. A larger study with a bigger sample size comparing the $\mathrm{r}$ ASRM system and Enzian system is required for further evaluation of disease.

\section{Conclusion:}

With all those observations it was concluded that, there was no relationship between frequency and severity of pain symptoms and disease stage of endometriosis. So a new classification system is warranted for management of endometriosis related pain. In view of these clinical results, use of the Enzian classification can be recommended as a supplement to the rASRM score for detailed description of endometriosis.

\section{References:}

1. Kennedy S, Bergqvist A, Chapron C, D'Hooghe T, Dunselman G, Greb R, Hummelshoj L, Prentice A, Saridogan et al. ESHRE guideline for the diagnosis and treatment of endometriosis. Hum Reprod. 2005 Oct; 20(10):2698-704.

2. Eskenazi B, Warner ML. Epidemiology of endometriosis. Obstet Gynecol Clin North Am. 1997 Jun; 24(2):235-58.

3. Nnoaham KE, Hummelshoj L, Webster P, D’Hooghe T, De Cicco Nardone F, De Cicco Nardone C, et al. Impact of endometriosis on quality of life and work productivity: a multicenter study across ten countries. Fertil Steril. 2011; 96(2):366-73. (35)

4. Propst AM, Laufer MR. Endometriosis in adolescents. Incidence, diagnosis and treatment. J Reprod Med. 1999; 44(9):751-8.

5. Yeung P, Jr, Sinervo K, Winer W, Albee RB., Jr. Complete laparoscopic excision of endometriosis in teenagers: is postoperative hormonal suppression necessary? Fertil Steril. 2011; 95(6):1909-12.

6. Porpora MG, Koninckx PR, Piazze J, Natili M, Colagrande S, Cosmi EV. Correlation between endometriosis and pelvic pain.J Am Assoc Gynecol Laparosc. 1999 Nov; 6(4):429-34.

7. De Graaff A, D’Hooghe TM, Dunselman GA, Dirksen CD, Hummelshoj L; WERF EndoCost Consortium, Simoens S. The significant effect of endometriosis on physical, mental and social wellbeing: results from an international cross-sectional survey. Hum Reprod. 2013 Oct; 28(10):2677-85.

8. Forman RG1, Robinson JN, Mehta Z, Barlow DH.Patient history as a simple predictor of pelvic pathology in sub fertile women. Hum Reprod. 1993 Jan; 8(1):53-5.

9. Eskenazi B, Warner M, Bonsignore L, Olive D, Samuels S, Vercellini P. Validation study of nonsurgical diagnosis of endometriosis.Fertil Steril. 2001 Nov; 76(5):929-35.

10. Hsu AL, Sinaii N, Segars J, Nieman LK, Stratton P. Relating pelvic pain location to surgical findings of endometriosis.Obstet Gynecol. 2011 Aug;118(2 Pt 1):223-30.

11. Meuleman C, Vandenabeele B, Fieuws S, Spiessens C, Timmerman D, D’Hooghe T. High prevalence of endometriosis in infertile women with normal ovulation and normospermic partners. Fertil Steril. 2009 Jul; 92(1):68-74.

12. Witz CA1, Burns WN. Endometriosis and infertility: is there a cause and effect relationship? Gynecol Obstet Invest. 2002; 53 Suppl 1:2-11.

13. Wykes CB, Clark TJ, Khan KS. Accuracy of laparoscopy in the diagnosis of endometriosis: a systematic quantitative review. BJOG. 2004 Nov; 111(11):1204-12.

14. Vercellini P, Trespidi L, De Giorgi O, Cortesi I, Parazzini F, Crosignani PG. Endometriosis and pelvic pain: relation to disease stage and localization.Fertil Steril. 1996 Feb; 65(2):299-304.

15. Porpora MG, Koninckx PR, Piazze J, Natili M, Colagrande S, Cosmi EV. Correlation between endometriosis and pelvic pain. J Am Assoc Gynecol Laparosc. 1999 Nov; 6(4):429-34.

16. Mehmud G, Akhtar T, Sadia S. Endometriosis: frequency and correlation between symptomatology and disease stage. J Coll Physicians Surg Pak. 2007 Apr; 17(4):199-202.

17. Haas $\mathrm{D}^{1}$, Oppelt P, Shebl O, Shamiyeh A, Schimetta W, Mayer R. Enzian classification: does it correlate with clinical symptoms and the rASRM score? Acta Obstet Gynecol Scand. 2013 May; 92(5):562-6. 